\title{
The neuroprotective effects of milk fat globule-EGF factor 8 against oligomeric amyloid $\beta$ toxicity
}

Endong Li ${ }^{\dagger}$, Mariko Noda ${ }^{\dagger}$, Yukiko Doi, Bijay Parajuli, Jun Kawanokuchi, Yoshifumi Sonobe, Hideyuki Takeuchi, Tetsuya Mizuno* and Akio Suzumura

\begin{abstract}
Background: Phosphatidylserine receptor is a key molecule that mediates the phagocytosis of apoptotic cells. Milk fat globule-EGF factor 8 (MFG-E8) is a phosphatidylserine receptor that is expressed on various macrophage lineage cells, including microglia in the central nervous system (CNS). Targeted clearance of degenerated neurons by microglia is essential to maintain healthy neural networks. We previously showed that the CX3C chemokine fractalkine is secreted from degenerated neurons and accelerates microglial clearance of neuronal debris via inducing the release of MFG-E8. However, the mechanisms by which microglia produce MFG-E8 and the precise functions of MFG-E8 are unknown.

Methods: The release of MFG-E8 from microglia treated with conditioned medium from neurons exposed to neurotoxic substances, glutamate or oligomeric amyloid $\beta$ (OAB) was measured by ELISA. The neuroprotective effects of MFG-E8 and MFG-E8 - induced microglial phagocytosis of oA $\beta$ were assessed by immunocytochemistry. The effects of MFG-E8 on the production of the anti-oxidative enzyme hemeoxygenase-1 (HO-1) were determined by ELISA and immunocytochemisty.
\end{abstract}

Results: MFG-E8 was induced in microglia treated with conditioned medium from neurons that had been exposed to neurotoxicants, glutamate or OAß. MFG-E8 significantly attenuated oAß-induced neuronal cell death in a primary neuron - microglia coculture system. Microglial phagocytosis of oA $\beta$ was accelerated by MFG-E8 treatment due to increased CD47 expression in the absence of neurotoxic molecule production, such as tumor necrosis factor-a, nitric oxide, and glutamate. MFG-E8 - treated microglia induced nuclear factor E(2) - related factor 2 (Nrf2) - mediated HO-1 production, which also contributed to neuroprotection.

Conclusions: These results suggest that microglia release MFG-E8 in response to signals from degenerated neurons and that MFG-E8 protects oA 3 -induced neuronal cell death by promoting microglial phagocytic activity and activating the Nrf2-HO-1 pathway. Thus, MFG-E8 may have novel roles as a neuroprotectant in neurodegenerative conditions.

Keywords: Microglia, Neuroprotection, MFG-E8, Nrf2

\section{Background}

Milk fat globule-EGF factor 8 (MFG-E8, lactadherin homolog in humans) is a phosphatidylserine (PS) receptor that acts as a cellular bridge between apoptotic cells and phagocytic cells and triggers the engulfment of cellular debris [1,2]. Recently, we showed that MFG-E8 is expressed in microglia and secreted in

\footnotetext{
* Correspondence: tmizuno@riem.nagoya-u.ac.jp

${ }^{\dagger}$ Equal contributors

Department of Neuroimmunology, Research Institute of Environmental Medicine, Nagoya University, Furo-cho, Chikusa-ku, Nagoya 464-8601, Japan
}

response to factors released from degenerated neurons, such as the CX3C chemokine fractalkine (FKN) [3]. FKN-induced MFG-E8 enhances microglial clearance of damaged neuronal debris. MFG-E8 has been shown to improve the pathogenesis of Alzheimer's disease (AD) by eliminating amyloid $\beta(A \beta)$ plaques [4]. In addition to microglia, MFG-E8 is also expressed in astrocytes, oligodendrocytes, and neurons [5], although it is uncertain what cell type predominantly produces MFG-E8. MFG-E8 is also implicated in the pathogenesis of immunerelated diseases in the periphery. MFG-E8 expression was 
reduced in lesions associated with murine experimental colitis, and MFG-E8 treatment markedly ameliorated disease progression [6]. This suggests that MFG-E8 acts as a suppressor of the peripheral immune system and that MFG-E8 may be a therapeutic target for immune-mediated bowel diseases $[7,8]$.

Microglia are resident immune cells in the central nervous system (CNS). In neurodegenerative diseases, such as AD and Parkinson's disease, microglia accumulate in lesions where they are thought to have both neurotoxic and neuroprotective functions [9]. In addition to producing various neuroprotective factors, microglia also phagocytose and remove injured neuronal debris or foreign antigens and this process is critically involved in maintaining healthy neuronal networks. However, the phagocytic activity of microglia is not only beneficial to neurons, but also exerts adverse effects by producing pro-inflammatory factors [10]. In the case of microglial A $\beta$ clearance, various cellular surface receptors are involved in recognizing and appropriately eliminating accumulated A $\beta$ plaques. These include CD14, CD36, CD47, CD200R, PS receptors, Toll-like receptors (TLRs), and receptors for advanced glycation end products (RAGE) [11,12]. Lipopolysaccharide (LPS) activates microglia and increases phagocytosis through the LPS receptor CD14, which may be involved in AD pathology $[13,14]$. Recent reports indicated that fibrillar $A \beta$ increases CD36 and CD47 expression in microglia [15], and CD36 is required for microglial phagocytosis of A $31-42$ [16]. CD47 is a key molecule in CNS inflammation that reduces macrophage activation and induces monocyte migration [17]. Intact myelin expresses CD47, which suppresses microglial phagocytosis of myelin [18]. Thus, CD47 seems to act as a "self" marker and inhibits excessive phagocytosis under normal conditions. On the other hand, $A \beta$ phagocytosis correlates with CD47 expression [19], which also suggests that CD47 plays a crucial role in target clearance during pathological conditions.

A $\beta$-induced oxidative stress is caused by reactive oxygen species (ROS) produced from microglia and astrocytes, and exacerbates the pathological conditions of $\mathrm{AD}[20,21]$. Induction of the nuclear factor $\mathrm{E}$ (2) - related factor 2 (Nrf2) signaling pathway could induce neuroprotection against some $A \beta$-related oxidative stressors.

In the present study, we show that MFG-E8 is released from microglia in response to various soluble factors (for example, FKN) released from degenerated neurons. MFGE8 increases microglial neuroprotective activity against oA $\beta$-induced neuronal cell death. This neuroprotection is mediated through enhanced microglial phagocytic activity via the CD47 pathway and activation of the Nrf2-HO-1 pathway.

\section{Materials and methods \\ Reagents}

L-glutamate and LPS were purchased from Sigma (St. Louis, MO, USA). MFG-E8 was obtained from R\&D Systems (Minneapolis, MN, USA). Tin (IV)-mesoporphyrin IX dichloride (SnMP), a specific inhibitor of $\mathrm{HO}-1$, was obtained from Frontier Scientific (Logan, UT, USA), and was dissolved in an arginine-containing solution as previously described [22,23].

\section{Preparation of oligomeric amyloid $\beta$ (oA $\beta$ ) solutions}

A $\beta 1-42$ solution was prepared as previously described [24]. Briefly, synthetic human A $\beta 1-42$ (Peptide institute, Osaka, Japan) was dissolved to $1 \mathrm{mM}$ in $100 \% 1,1,1,3,3,3$ hexafluoro-2-propanol (HFIP). HFIP was dried using a vacuum desiccator and resuspended to a concentration of $5 \mathrm{mM}$ in DMSO. To form oligomers, amyloid peptide was diluted to a final concentration of $100 \mu \mathrm{M}$ with Ham's F-12, incubated at $4^{\circ} \mathrm{C}$ for $24 \mathrm{~h}$, and then immediately added to cultures at a final concentration of $5 \mu \mathrm{M}$. Formation of oA $\beta$ was confirmed using western blot analysis as previously described [24].

\section{Cell culture}

All animal protocols were approved by the Animal Experiment Committee of Nagoya University. Primary neuronal cultures were prepared from the cortices of C57BL/6 mouse embryos at embryonic Day 17 (E17) as previously described [25]. Briefly, cortical fragments were dissociated into single cells in dissociation solution (Sumitomo Bakelite, Akita, Japan), and resuspended in neuron culture medium (Sumitomo Bakelite). Neurons were seeded onto 12-mm polyethyleneimine-coated glass coverslips (Asahi Techno Glass Corp., Chiba, Japan) at a density of $5.0 \times 10^{4}$ cells/well in 24-well plates and incubated at $37^{\circ} \mathrm{C}$ in a humidified atmosphere containing $5 \%$ $\mathrm{CO}_{2}$. The purity of the cultures was more than $95 \%$ as determined by NeuN-specific immunostaining.

Microglia were isolated from primary mixed glial cell cultures prepared from newborn C57BL/6 mice at Day in vitro (DIV) 14 using the "shaking off" method, as previously described [26]. The purity of the cultures was 97 to $100 \%$ as determined by immunostaining for the Fc receptor. Cultures were maintained in Dulbecco's modified Eagle medium supplemented with $10 \%$ fetal calf serum, $5 \mu \mathrm{g} / \mathrm{mL}$ bovine insulin and $0.2 \%$ glucose. Microglia were seeded at a density of $7.0 \times 10^{4}$ or $1.0 \times 10^{5}$ cells/well in 96 - or 48 -well plates, respectively.

Neuron-microglia co-cultures were prepared by adding $1.0 \times 10^{5}$ microglia in $100 \mu \mathrm{L}$ neuronal medium to neuronal cultures $\left(5.0 \times 10^{4}\right.$ neuronal cells $)$ on DIV 14 in 24-well plates. The cultures were maintained in neuron culture medium. 


\section{Measurement of MFG-E8 levels}

MFG-E8 secreted from mouse primary microglia or cortical neurons was measured using an ELISA (R\&D Systems, Minneapolis, MN, USA) according to the manufacturer's instructions. Neurons and microglia were treated with oA $\beta(5 \mu \mathrm{M})$ or L-glutamate $(20 \mu \mathrm{M})$ for $24 \mathrm{~h}$ at $37^{\circ} \mathrm{C}$. In addition, neuronal conditioned medium (Neu $\mathrm{CM}$ ) was prepared as follows: $5.0 \times 10^{4}$ neuronal cells in neuronal medium were treated with oA $\beta(5 \mu \mathrm{M})$ or L-glutamate $(20 \mu \mathrm{M})$ for $24 \mathrm{~h}$, and the supernatant was collected. A total of $1.0 \times 10^{5}$ microglia were treated with Neu CM for 24 h, and then MFG-E8 in the supernatant was measured.

\section{RT-PCR}

Total RNA was extracted from microglia and neurons using an RNeasy Mini Kit (Qiagen, Tokyo, Japan). A firststrand cDNA library was obtained using SuperScript II (Invitrogen, Carlsbad, CA, USA) and oligo (dT) ${ }_{12-18}$ (Invitrogen) as the first-strand primer. Negative control reactions were performed using the same system after heat denaturing the reverse transcriptase. Transcripts encoding mouse CD36, CD47, and glyceraldehydes-3phosphate dehydrogenase (GAPDH) were amplified by RT-PCR using $0.1 \mu \mathrm{g}$ of first-strand cDNA, Blend Taq polymerase (Toyobo Co., Osaka, Japan), and oligonucleotide primers (Table 1$)$.

\section{Immunocytochemistry}

Cells were fixed with $4 \%$ paraformaldehyde for 15 minutes at room temperature, blocked with $5 \%$ serum, and permeabilized with $0.05 \%$ Triton X-100. Neurons were stained with mouse polyclonal anti-MAP-2 antibodies (1:1,000; Chemicon, Temecula, CA, USA) and Alexa 488-conjugated secondary antibodies (1:1,000; Invitrogen). Microglia were stained with Cy5-conjugated rat anti-mouse CD11b monoclonal antibodies (1:300; BD Biosciences, Franklin

Table 1 Oligonucleotide primers of CD14, CD36, CD47 and GAPDH

\begin{tabular}{llc}
\hline Gene & Sequence $\left(\mathbf{5}^{\prime}\right.$ to $\left.\mathbf{3}^{\prime}\right)$ & Expected size (bp) \\
\hline CD14 & & \\
\hline Sense & 5'-GACCATGGAGCGTGTGCTTG & 464 \\
\hline Antisense & 5'-GCCACTGCTGCAGTTCTGCGAG & \\
\hline CD36 & & \\
\hline Sense & 5'-GCAAAGAAGGAAAGCCTGTG & 367 \\
\hline Antisense & 5'-ATCACCACTCCAATCCCAAG & \\
\hline CD47 & & \\
\hline Sense & 5'-CTGGTGCTCACAGTCATCGT & \\
\hline Antisense & 5'-GACCAAAGCAAGGACGTAGC & \\
\hline GAPDH & & \\
\hline Sense & 5'-ACTCACGGCAAATTCAACG & \\
\hline Antisense & 5'-CCCTGTTGCTGTAGCCGTA & \\
\hline
\end{tabular}

Lakes, NJ, USA) prior to fixation. Images were analyzed using a deconvolution fluorescence microscope system (BZ-8000; Keyence Corporation, Osaka, Japan).

Surviving neurons were identified based on their cytoskeletons as previously described [25]. Viable neurons stained strongly with an anti-MAP-2 antibody, whereas damaged neurons showed weaker staining. MAP-2 - positive neurons were counted in representative areas in each well. In five independent experiments, more than 200 neurons were evaluated in each well by a scorer who was blind to the experimental conditions. The number of viable neurons in untreated cultures was set to $100 \%$.

\section{Evaluation of microglial phagocytosis of $\mathrm{OA} \beta$}

Microglia were seeded at a density of $6.0 \times 10^{4}$ cells/well in a poly-L-lysine-coated eight-well chamber plate. Cells were pre-treated with MFG-E8 (1 to $100 \mathrm{ng} / \mathrm{mL}$ ) for $1 \mathrm{~h}$, and then oA $\beta(5 \mu \mathrm{M})$ was added to the culture for $6 \mathrm{~h}$. The cells were subsequently fixed in $4 \%$ paraformaldehyde. Microglia were stained with a rabbit polyclonal anti-Rab7 antibody (1:200; Cell Signaling Technology, Danvers, MA, USA) and Alexa 568-conjugated secondary antibody (1:1,000; Invitrogen). oA $\beta$ was stained with a mouse polyclonal anti-4 G8 antibody (1:1000; Chemicon,) and Alexa 488 - conjugated secondary antibody (1:1,000; Invitrogen). Immunostained images were observed under confocal laser scanning microscope (LSM 710; ZEISS, Jena, Germany).

\section{Fluorescence-activated cell sorting (FACS) analysis of cell surface antigen expression}

Microglia were seeded at a density of $1.0 \times 10^{6}$ cells/dish in a 6-cm dish. Cells were treated with $100 \mathrm{ng} / \mathrm{mL}$ MFGE8 for $72 \mathrm{~h}$, and then immunostaining was performed using standard procedures. The primary antibodies included anti-CD36 (1:200; BD Biosciences) and antiCD47 (1:200; BD Biosciences) and the secondary antibodies were conjugated to FITC (1:1,000; BD Biosciences).

\section{Measurement of HO-1, tumor necrosis factor-a (TNF-a), nitric oxide (NO), and glutamate}

Microglia were treated with LPS or MFG-E8 (1 to $100 \mathrm{ng} / \mathrm{mL}$ ) and $\mathrm{oA} \beta$ for $48 \mathrm{~h}$. The supernatants were assessed using a TNF- $\alpha$ ELISA (BD Pharmingen, Franklin Lakes, NJ, USA). Cells were lysed in extraction buffer (1\% NP-40 in PBS) and assayed for HO-1 with an ELISA (Takara Bio, Mie, Japan). The NO levels were determined using the Griess reaction as previously reported [27]. To measure glutamate levels, a colorimetric assay kit (Yamasa Corporation, Tokyo, Japan) was used as previously described [28].

\section{Statistical analysis}

Statistically significant differences between the experimental groups were determined by one-way ANOVA 
followed by Dunnett's or Tukey's tests for multiple comparisons. Statistical analyses were performed using Prism 4 for Windows (GraphPad Software, San Diego, CA, USA). P-values less than 0.05 were considered significant.

\section{Results}

Microglia secrete MFG-E8 in response to factors released from degenerated neurons

We first examined whether microglia produce MFG-E8 in response to soluble factors released from degenerated neurons. The direct glutamate or oA $\beta$ treatment did not induce MFG-E8 secretion from either microglia or neurons. The MFG-E8 levels in the culture medium for neurons or microglia were negligible (Figure 1A). However, the levels of MFG-E8 secreted from microglia significantly increased when the cells were treated with conditioned medium from neurons that had been exposed to glutamate or oA $\beta$ (Figure 1A). The Western blot analysis for oA $\beta$ used in the experiments was shown in Figure 1B. Neurons released significant amounts of soluble fractalkine (sFKN) when exposed to oA $\beta$ as well as glutamate (Figure $1 \mathrm{C}$ ). In a previous study, we showed that sFKN is secreted from glutamate-damaged neurons and induces MFG-E8 expression in microglia [3], suggesting that sFKN may play a role as a mediator to induce MFG-E8 release from microglia.
A

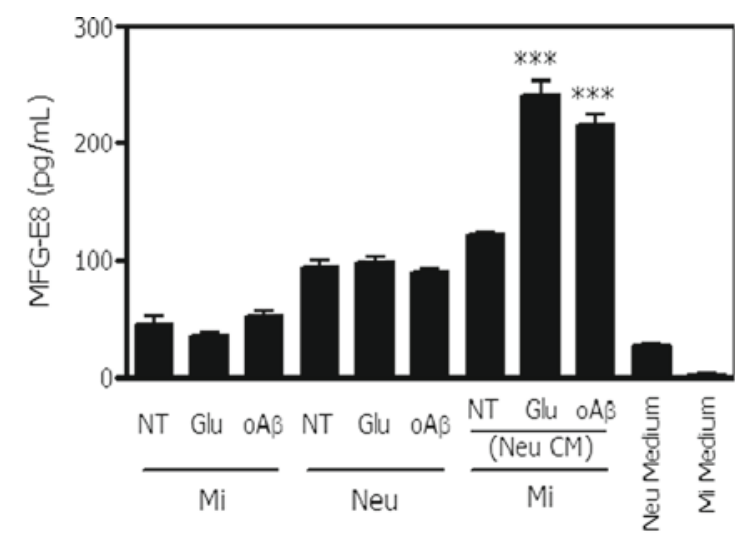

C

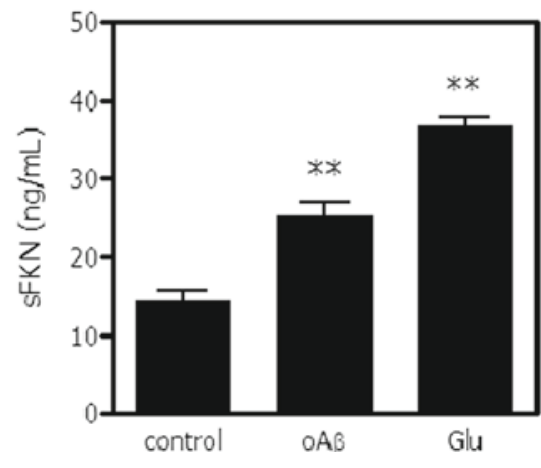

B

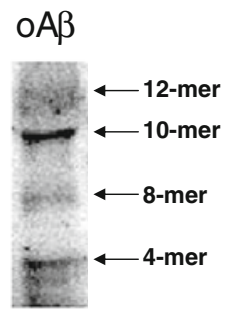

Figure 1 Induction of MFG-E8 by soluble factors released from degenerated neurons. (A) The levels of MFG-E8 secreted from microglia (Mi) and cortical neurons ( $\mathrm{Neu}$ ) treated with $20 \mu \mathrm{M}$ glutamate (Glu) or $5 \mu \mathrm{M}$ oligomeric amyloid $\beta$ (oAB) were measured by ELISA. In addition, MFG-E8 released from microglia exposed to neuronal conditioned medium (Neu CM) that had been treated with Glu or oA $\beta$ was also measured. Results are presented as the means with S.E.M. $(n=3)$. Glu- or oA -treated neuronal conditioned medium significantly induced MFG-E8 expression from microglia compared with the untreated control samples. ${ }^{* *}: P<0.001$ (one-way ANOVA with Dunnett's post-hoc test). (B) The Western blot data of OA $\beta$ used in the present study. The blot was incubated in mouse anti-A $\beta$ monoclonal antibody $(6 E 10)(1: 1,000$, Chemicon). (C) The levels of the soluble secreted form of fractalkine (sFKN) released from cortical neurons treated with $20 \mu \mathrm{M}$ glutamate (Glu) or $5 \mu \mathrm{M}$ oligomeric amyloid $\beta$ (OA $\beta$ ) were measured. The results are presented as the means with S.E.M. $(n=3)$. Glu and oA $\beta$ treatment significantly induced sFKN release from neurons compared to the untreated control samples. ${ }^{* *}: P<0.01$ (one-way ANOVA with Dunnett's post-hoc test). 

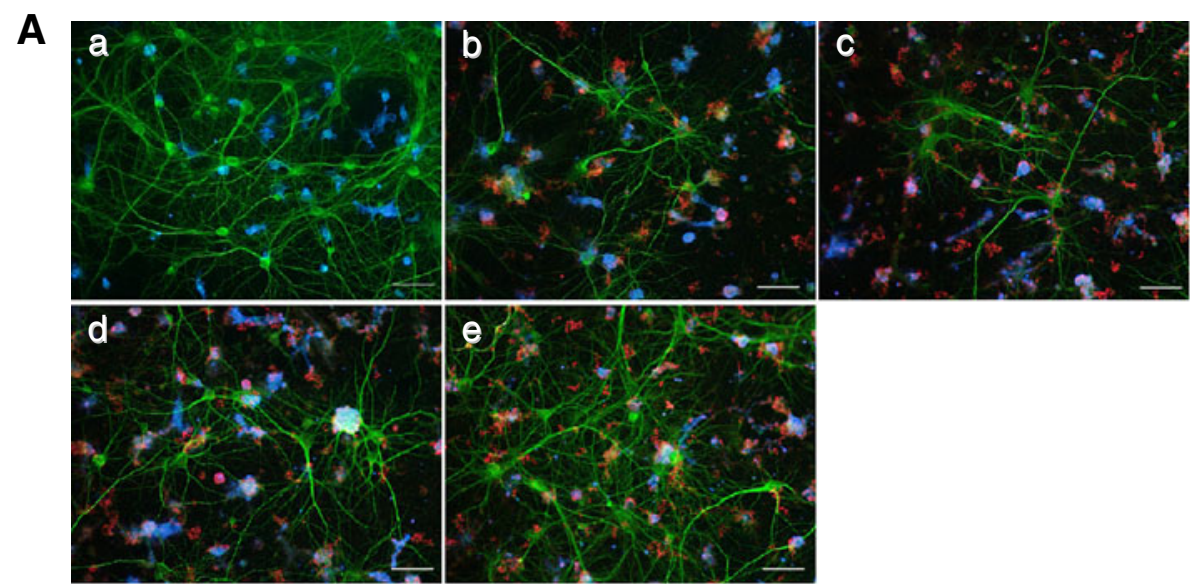

B
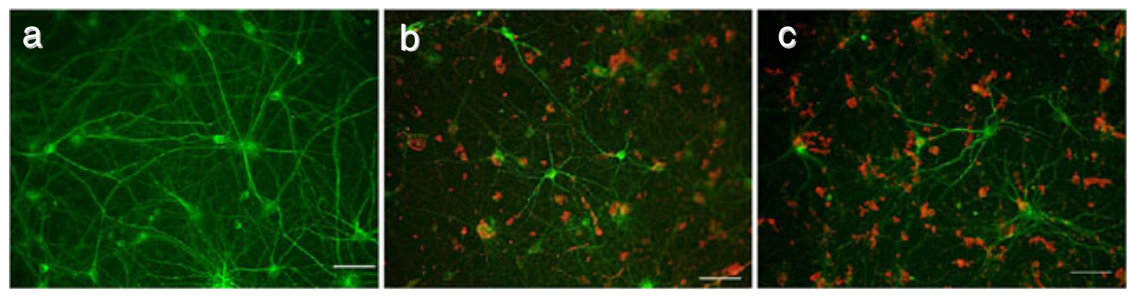

C

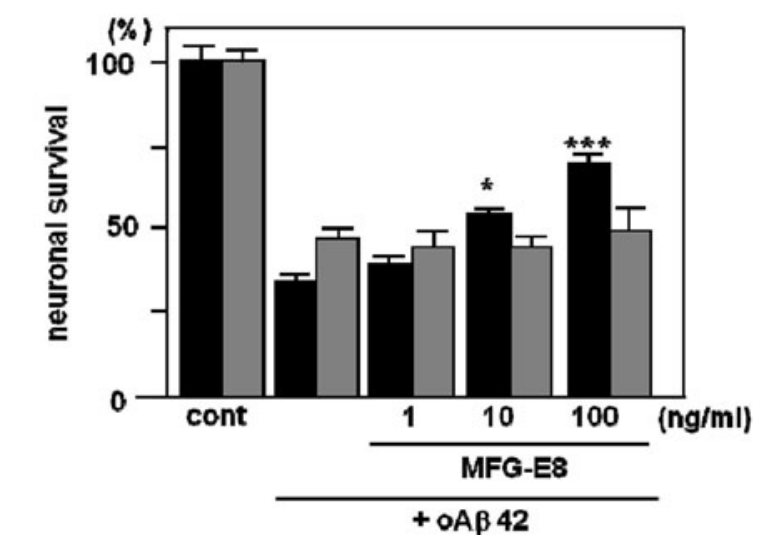

Neuron microglia co-cultures Neuronal cultures

Figure 2 MFG-E8 exerts neuroprotective effects in the presence of microglia. The effect of MFG-E8 treatment against oA neuron - microglia co-cultures (A) and neuronal cultures (B). Neurons were stained with an anti-MAP-2 antibody (green), microglia were stained with a Cy5-conjugated anti-CD11b antibody (blue), and oA $\beta$ was stained with an anti-4 G8 antibody (red). Scale bar $=50 \mu \mathrm{m}$. Untreated neuronal cultures and neuron-microglia cocultures (1:2 neurons to microglia) (cont; $a$ ). Five micromolar of oA $\beta$ induced neuronal loss in both neuronal and neuron-microglia cocultures (b). In the presence of microglia, MFG-E8 treatment induced neuroprotective effects in a dose-dependent manner (doses were $1 \mathrm{ng} / \mathrm{mL}(c), 10 \mathrm{ng} / \mathrm{mL}(d), 100 \mathrm{ng} / \mathrm{mL}(e)$ in (A)). On the other hand, MFG-E8 did not show any effects on oA 3 -induced neurotoxicity, even at the highest dose $(100 \mathrm{ng} / \mathrm{mL} ; \mathrm{C}$ in (B)). (C) Neuronal survival was estimated as the percentage of intact neurons in the treated sample relative to the untreated sample. The results are presented as the means with S.E.M. $(n=3)$, in which 10 randomly selected fields were analyzed. Significant differences compared to samples only treated with oA $\beta$ are noted. ${ }^{*}: P<0.05,{ }^{* * *}: P<0.001$, (one-way ANOVA with Dunnett's post-hoc test).

\section{MFG-E8 directly induces microglial neuroprotective} effects

We then examined the direct effects of MFG-E8 on neuronal survival. There has been little evidence indicating that MFG-E8 exerts neuroprotective effects, aside from our previous report in which neutralizing MFG-E8 markedly attenuated sFKN-induced neuroprotection [3]. Therefore, we first determined whether MFG-E8 has direct neuroprotective effects against oA $\beta$ toxicity in neuron-microglia cocultures (Figure $2 \mathrm{~A} a-e$ ) or in 
A
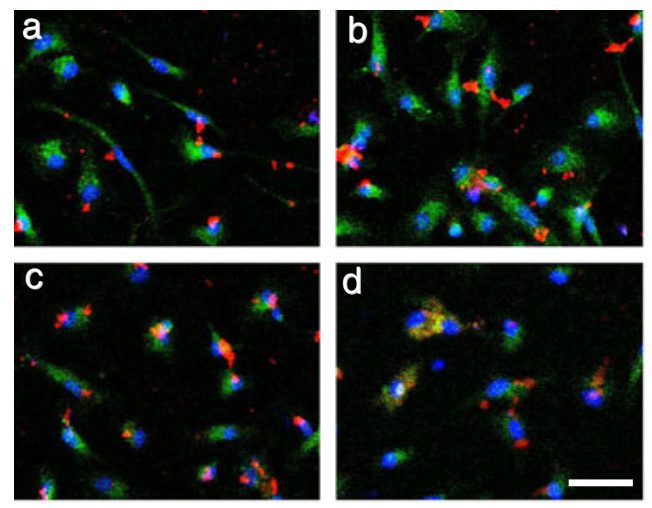

C

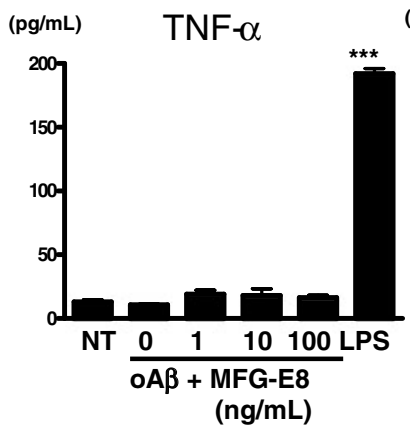

$\mathbf{F}$

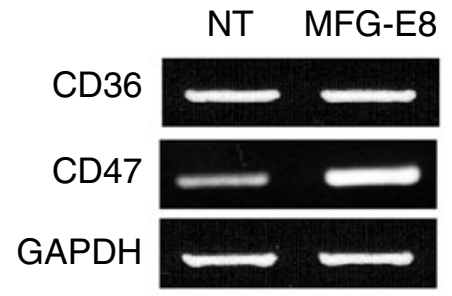

B

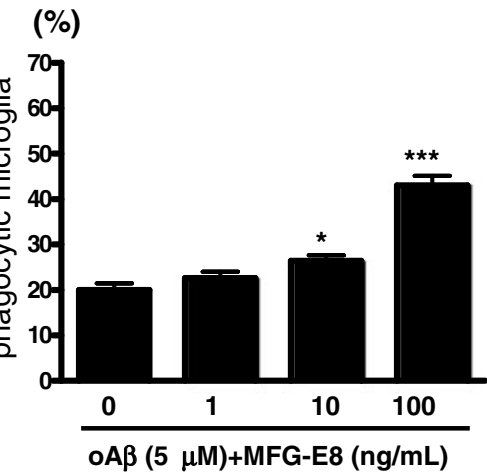

D E

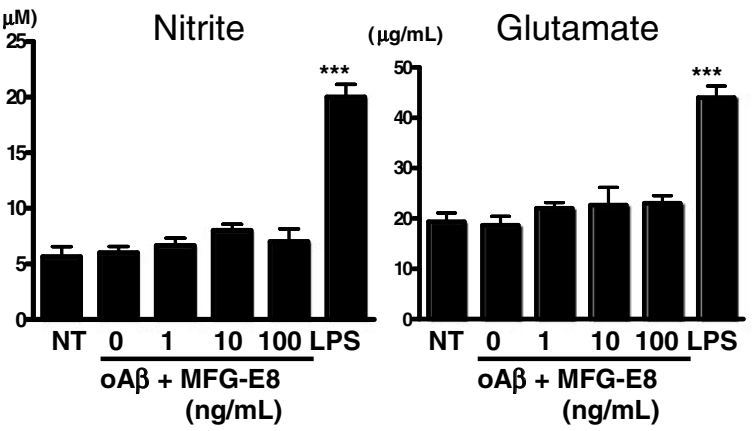

G

CD36 CD47 .

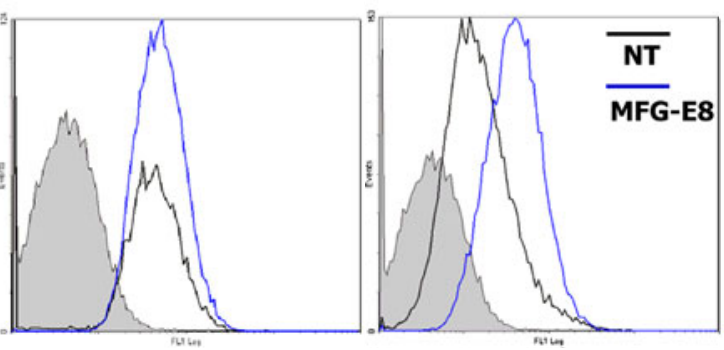

Figure 3 MFG-E8 induces microglial phagocytosis of oA $\beta$. (A) Microglia were pre-treated with $1 \mathrm{ng} / \mathrm{mL}$ (b), $10 \mathrm{ng} / \mathrm{mL}$ (c) or $100 \mathrm{ng} / \mathrm{mL}(d)$ MFG-E8 for $1 \mathrm{~h}$, and then $5 \mu \mathrm{M} \circ \mathrm{A} \beta$ was added to the culture for $6 \mathrm{~h}$. Primary antibodies recognizing Rab7 (green), oA $($ red) and nuclei (b/ue) were used $(a-d)$. A few OAB-incorporated microglia were detected in the absence of MFG-E8 $(a)$, while a sufficient number of microglia that had engulfed $\mathrm{OA} \beta$ were detected in the presence of the higher MFG-E8 dose $(d)$ under confocal laser scanning microscope. Scale bar $=50 \mu \mathrm{m}$. (B) Quantification of the phagocytosis index, which is defined as the percentage of total microglial-Rab7 staining (green) that overlaps with oA staining (red). The results are presented as the means with S.E.M. $(n=3)$, in which 10 randomly selected fields were analyzed. Significant differences compared with samples that were only treated with oA $(\mathrm{OA} \beta$ ) are noted. *: $P<0.05$ (one-way ANOVA with Dunnett's post-hoc test). The measurement of TNF-a (C), nitrite (D), and glutamate (E) produced by microglia treated with MFG-E8 plus oA 3 was performed. After $24 \mathrm{~h}$ treatment with $1 \mathrm{ng} / \mathrm{mL}, 10 \mathrm{ng} / \mathrm{mL}$ or $100 \mathrm{ng} / \mathrm{mL}$ MFG-E8 with $5 \mu \mathrm{M}$ oA $\beta$, the supernatants of microglial cultures were analyzed. $100 \mathrm{ng} / \mathrm{mL}$ LPS treatment for $24 \mathrm{~h}$ was used as a positive control. The results are presented as the means with S.E.M. $(n=5)$. Significant differences compared to untreated microglia (NT) are noted. ***: $P<0.001$, (one-way ANOVA with Dunnett's post-hoc test). (F) mRNA expression levels of oA $\beta$ phagocytosis-related cellular membrane surface antigens, CD36 and CD47, in microglia treated with $100 \mathrm{ng} / \mathrm{mL}$ MFG-E8 for $24 \mathrm{~h}$ was assessed using RT-PCR. GAPDH expression was used as a control. (D) CD36 and CD47 protein levels were assessed by FACS analysis. Microglia were treated with $100 \mathrm{ng} / \mathrm{mL}$ MFG-E8 for $72 \mathrm{~h}$. The gray-filled curve represents the isotype-matched control. The black and blue lines indicate untreated (NT) and MFG-E8-treated samples, respectively. 
A
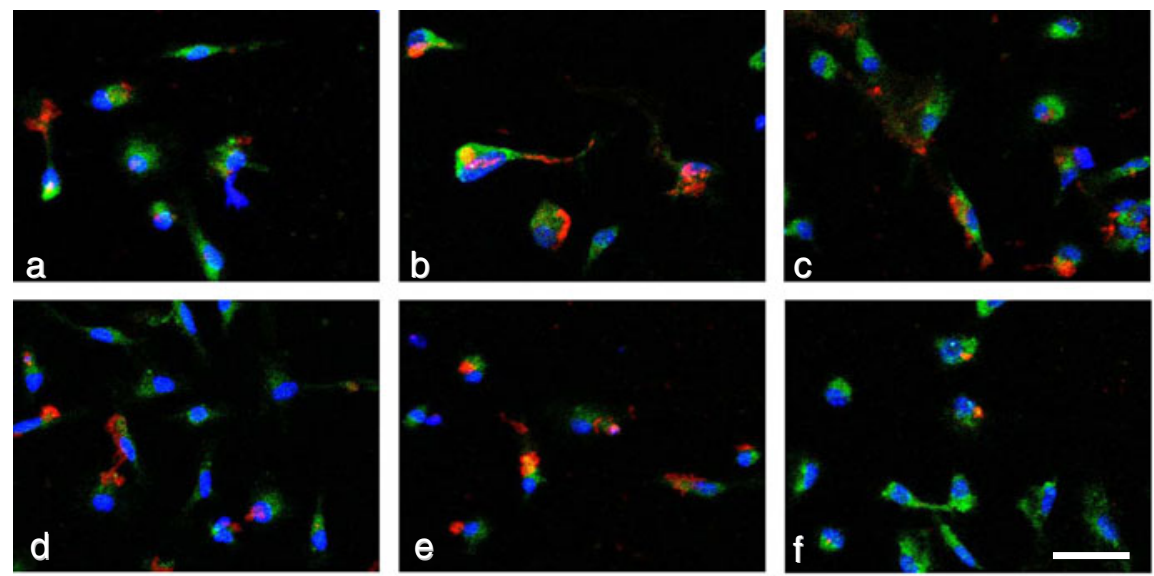

B

(\%)

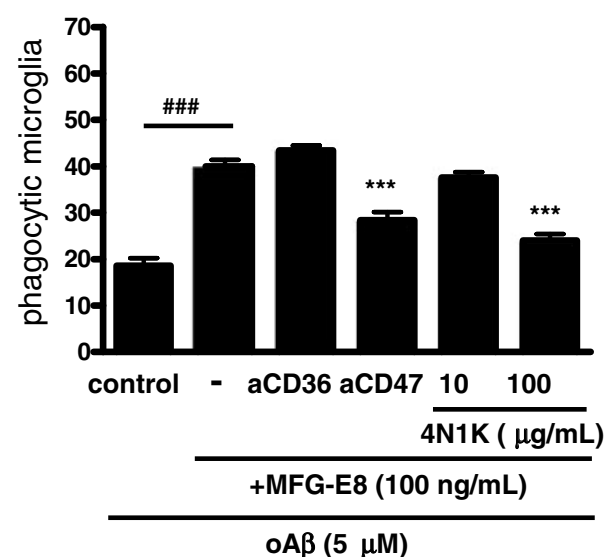

C

(\%)

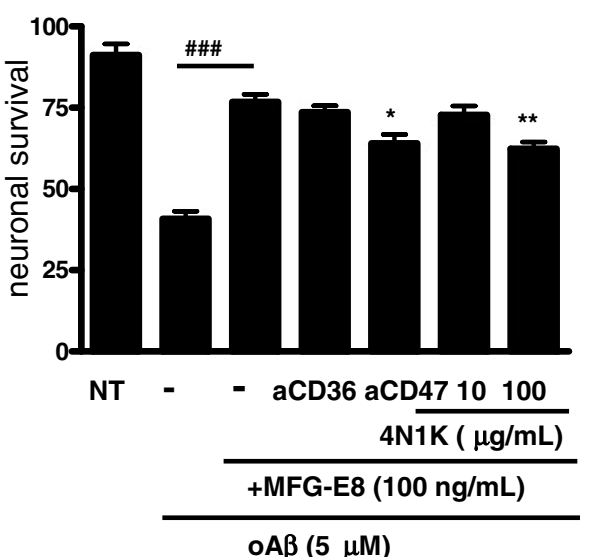

Figure 4 MFG-E8 enhances microglial phagocytosis of oA $\beta$ through CD47. (A) Microglia were treated with $1 \mu \mathrm{g} / \mathrm{mL}$ of isotype-matched control $\operatorname{lgG}(a, b), 100 \mathrm{ng} / \mathrm{mL}$ MFG-E8 $(b-f), 1 \mu \mathrm{g} / \mathrm{mL}$ of anti-CD36 neutralizing antibody $(c), 1 \mu \mathrm{g} / \mathrm{mL}$ of anti-CD47 neutralizing antibody (d), the peptide antagonist $4 \mathrm{~N} 1 \mathrm{~K} 10 \mu \mathrm{g} / \mathrm{mL}$ (e) or $100 \mu \mathrm{g} / \mathrm{mL}(f)$ for $1 \mathrm{~h}$, and then $5 \mu \mathrm{M}$ oA was added to the culture for 24 h. Primary antibodies against Rab7 (green), oA $\beta$ (red) and nuclei (blue) were used $(a-f)$. Scale bar $=50 \mu \mathrm{m}$. (B) Quantification of the phagocytosis index. Results show the means with S.E.M. $(n=3)$, in which 10 randomly selected fields were analyzed. Significant differences compared with the isotype-matched control samples (\#) or samples treated only with MFG-E8 (*) are noted. ***: $P<0.001$; \#\#\#: $P<0.001$ (one-way ANOVA with Tukey's post-hoc test). (C) The effects of blockade of CD36 and CD47 in MFG-E8-treated microglia on neuronal survival. Neuron - microglia co-cultures were pre-treated with MFG-E8 for $1 \mathrm{~h}$ in the presence of anti-CD36 neutralizing antibody, anti-CD47 neutralizing antibody, and $10 \mu \mathrm{g} / \mathrm{mL}$ or $100 \mu \mathrm{g} / \mathrm{mL} 4 \mathrm{~N} 1 \mathrm{~K}$. Then, the cultures were treated with oA $\beta$ for 24 h. The neuronal survival rate was estimated. The results are presented as the means with S.E.M. ( $n=5)$, in which 10 randomly selected fields were analyzed. Significant differences compared with the isotype-matched control samples (\#) or samples treated only with MFG-E8 (*) are noted. *: $P<0.05 ;$ \#\#\#: $P<0.001$ (one-way ANOVA with Tukey's post-hoc test).

neuronal cultures (Figure $2 \mathrm{~B} a-c$ ). Treating neurons with $5 \mu \mathrm{M}$ oA $\beta$ for $24 \mathrm{~h}$ induced obvious cell death in both neuron - microglia cocultures (Figure $2 \mathrm{~A} b, \mathrm{C}$ ) and neuron cultures (Figure 2Bb, C). MFG-E8 significantly inhibited oA $\beta$-induced cell death in a dose-dependent manner in neuron - microglia cocultures (Figure 2Ac-e, $\mathrm{C}$ ), but not in neuron cultures (Figure $2 \mathrm{~B} c, \mathrm{C}$ ).
MFG-E8 increases phagocytosis of oA $\beta$ through a CD47-mediated signaling pathway in microglia

To explore the detailed mechanisms by which MFG-E8 exerts neuroprotection in the presence of microglia, we investigated whether MFG-E8 increased microglial phagocytosis of $A \beta$ under confocal laser scanning microscope. Non-treated microglia minimally engulfed 
A

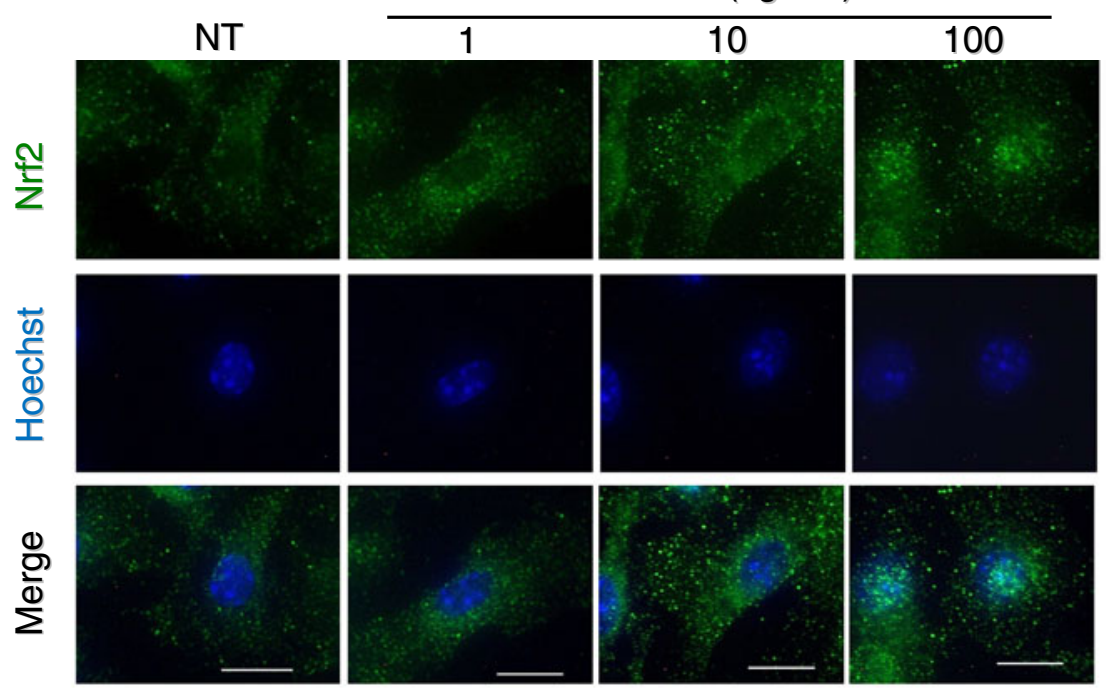

B

C
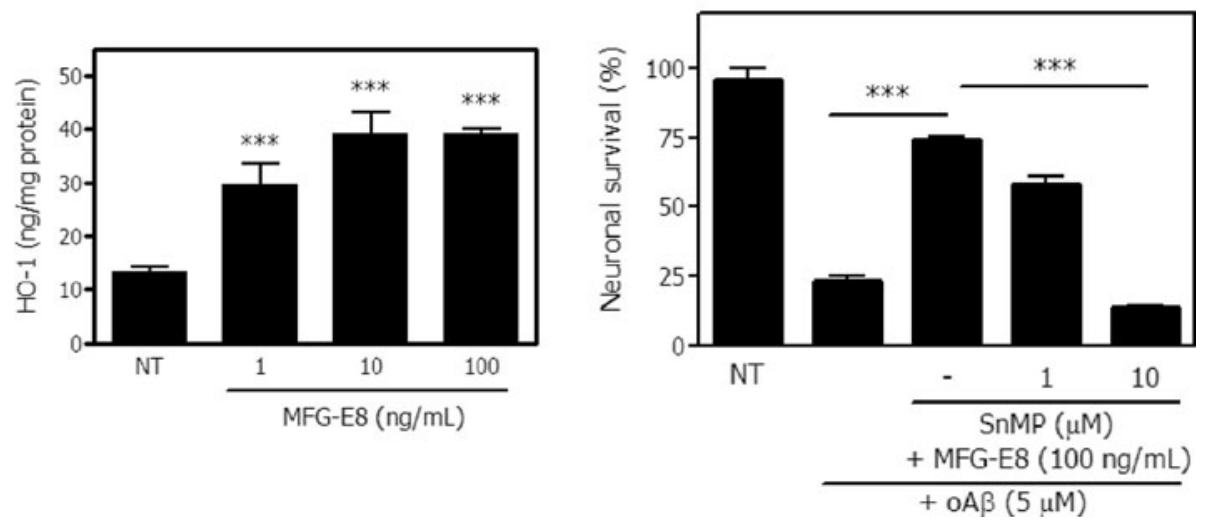

Figure 5 MFG-E8 exerts neuroprotective effects by activating the Nrf-2-HO-1 pathway in microglia. (A) Immunofluorescence images of Nrf2 (green) and nuclei (Hoechst; blue) in microglia treated with the indicated concentrations of MFG-E8. Scale bar $=10 \mu \mathrm{m}$. (B) After microglia were treated with the indicated concentrations of MFG-E8 for 24 h, HO-1 protein expression levels were measured by ELISA. Significant differences compared with untreated samples $\left(^{*}\right)$ are noted. ${ }^{* *}: P<0.001$ (one-way ANOVA with Dunnett's post-hoc test). (C) Neuron - microglia co-cultures were pre-treated with MFG-E8 for $3 \mathrm{~h}$ in the presence of the HO-1 inhibitor SnMP. Then, the cultures were treated with oA $\mathrm{A}$ for $24 \mathrm{~h}$ The neuronal survival rate in the presence of MFG-E8 and SnMP was estimated. The results are presented as the means with S.E.M. ( $n=3$ ), in which 10 randomly selected fields were analyzed. Significant differences compared with oA $\beta$ plus MFG-E8 treated samples are noted. ***: $P$ $<0.001$ (one-way ANOVA with Dunnett's post-hoc test).

$\mathrm{A} \beta$, as determined by the co-localization of the phagosome maturation marker Rab-7 and $\mathrm{A} \beta$ (Figure $3 \mathrm{~A} a$ ). However, MFG-E8 increased microglial phagocytosis of $A \beta$ in a dosedependent manner (Figure $3 \mathrm{~A} b-d$ ). The ratio of phagocytic to total cells is shown graphically (Figure 3B) and treating with $10 \mathrm{ng} / \mathrm{mL}$ and $100 \mathrm{ng} / \mathrm{mL}$ MFG-E8 significantly increased this ratio. The neurotoxic molecules, such as TNF- $\alpha$, NO, and glutamate, were not induced by MFGE8- induced microglial phagocytosis of $A \beta$ (Figure 3C-E).
We next examined the effects of MFG-E8 on the expression of principal phagocytosis-related surface receptors in microglia. The expression of the classical microglial phagocytosis receptors CD14 and TLR4, which are LPS receptors, was unchanged with MFG-E8 treatment, and TLR6 and TLR9 expression was also unchanged (data not shown). In AD brains, microglia reportedly interact with amyloid plaque by forming functional receptors including $\alpha_{6} \beta_{1}$ integrin, CD36 and 


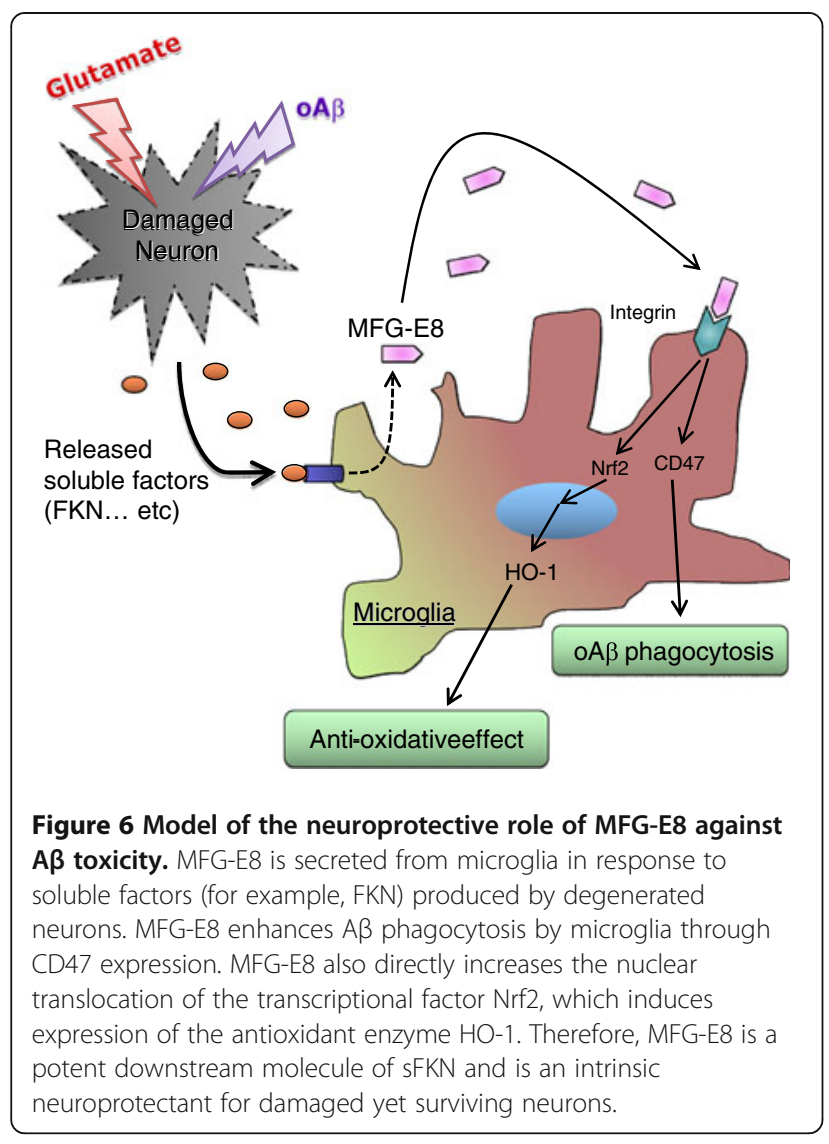

CD47, which act concertedly to engulf $A \beta[19,29]$. Thus, we next examined the effect of MFG-E8 on CD36 and CD47 expression in microglia. Treating microglia with $100 \mathrm{ng} / \mathrm{mL}$ of MFG-E8 markedly induced CD47 mRNA expression but not CD36 expression (Figure 3F). FACS analysis for CD36 and CD47 protein levels yielded similar results; treating microglia with MFG-E8 for $72 \mathrm{~h}$ increased microglial CD47 expression, but not CD36 expression (Figure 3G).

Then, we examined the involvement of these cellular surface antigens in $A \beta$ phagocytosis. MFG-E8 $(100 \mathrm{ng} / \mathrm{mL})$ increased phagocytosis almost two-fold relative to the unstimulated control (Figure $4 \mathrm{~A} b, \mathrm{~B}$ ). Although blocking CD36 with a specific antibody did not have an effect (Figure 4Ac, B), blocking CD47 with a specific antibody completely suppressed MFGE8 - induced phagocytosis (Figure 4Ad, B). High concentrations of $4 \mathrm{~N} 1 \mathrm{~K}$, a CD47 blocking peptide [30], similarly inhibited phagocytosis (Figure 4Ae, $f, \mathrm{~B}$ ). These data suggest that $A \beta$ was recognized and phagocytosed by microglia through a CD47-mediated pathway. Moreover, we examined whether blockade of CD47 affects neuronal survival by MFG-E8-treated microglia. We confirmed that blockade of CD47 with anti-CD47 antibody or $4 \mathrm{~N} 1 \mathrm{~K}$ decreased neuronal survival (Figure 4C).

\section{MFG-E8 induces neuroprotective effects in microglia through the Nrf2-HO-1 pathway}

We previously showed that neuroprotection by sFKN is initiated by the nuclear translocation of the transcription factor Nrf2 and subsequent HO-1 production [3]. Thus, we examined whether MFG-E8 induces a change in the subcellular localization of Nrf2. MFG-E8 markedly enhanced the nuclear translocation of Nrf2 in a dose-dependent manner, as determined by immunostaining (Figure 5A). In addition, MFG-E8 also dosedependently increased $\mathrm{HO}-1$ production (Figure $5 \mathrm{~B}$ ). However, the expression of another Nrf2-induced phase-II enzyme, NAD(P)H quinine oxidoreductase-1 (NQO-1), was not changed by MFG-E8 (data not shown). Finally, we examined the role of $\mathrm{HO}-1$ in MFG-E8-induced neuroprotection against $A \beta$ toxicity. The neuroprotective effects of MFG-E8 were abolished by treating with $10 \mu \mathrm{M}$ SnMP, an HO-1 inhibitor, although a lower concentration of SnMP did not exert these effects (Figure $5 \mathrm{C}$ ). We also examined the expression of neuroprotectants other than $\mathrm{HO}-1$ and NQO-1. MFG-E8 did not affect the mRNA levels of BDNF, GDNF, NGF, TGF- $\beta$ or IL-10 (data not shown ). Therefore, Nrf2-induced HO-1 production by microglia may contribute to the neuroprotective effects of MFG-E8.

\section{Discussion}

In this study, we showed for the first time that MFG-E8 has neuroprotective effects against oA $\beta$ toxicity by enhancing microglial phagocytic activity and exerting anti-oxidant effects. MFG-E8 was initially described as a mouse mammary epithelial cell surface protein but was more recently identified as a glycoprotein secreted from macrophages in germinal centers of the spleen and lymph nodes [1]. Phagocytosis is thought to be induced by "eat-me" signals, such as the nucleotide GDP and PS. MFG-E8 is a PS receptor and promotes apoptotic cell clearance [31]. In the present study, it is clear that MFG-E8 also plays a role in the engulfment of $A \beta$ by microglia. It has been shown that MFG-E8 enhances phagocytosis of UV-treated apoptotic neurons by microglia [32], and that a MFG-E8/lactadherin deficiency markedly reduces $A \beta$ phagocytosis by macrophages [4]. We also revealed that microglial phagocytosis of $A \beta$ is mediated through CD47 expressed on microglia. Other microglial phagocytosis-related receptors, such as CD14, and components of the senile plaqueinteracted receptor, such as CD36, were not affected by MFG-E8. Although both CD14 and CD36 have been associated with MFG-E8 [33] or microglial A $\beta$ phagocytosis [16], they were not induced by MFG-E8 treatment in this study. Blocking CD47 with a neutralizing antibody or the $4 \mathrm{~N} 1 \mathrm{~K}$ peptide significantly reduced $\mathrm{A} \beta$ 
phagocytosis. The CD47 ligand is a signal regulatory protein- $\alpha$ (SIRP $\alpha)$ that is expressed on the cellular membrane of neurons as well as glial cells [17]. SIRP $\alpha$ is also reportedly a negative regulator of the CD47-mediated signaling pathway $[17,18]$. We previously showed that MFGE8 expression is induced by sFKN released from degenerated neurons [3]. sFKN increased HO-1 production via the JNK MAPK-Nrf2 pathway in microglia and exerted neuroprotection against excitotoxicity. In this study, MFG-E8 increased the nuclear translocation of Nrf2 and enhanced HO-1 production in microglia. Since the expression of another Nrf2-induced anti-oxidative enzyme, NQO-1, was not increased by MFG-E8, HO-1 might mediate MFG-E8 - induced neuroprotection against $A \beta$ toxicity. Accordingly, MFG-E8 may be downstream of sFKN in microglia. A $\beta$-induced oxidative stress is mediated through ROS production by glial cells and also by neurons [20,21,34-36]. ROS may lead to neuronal degeneration in AD. These results delineate a novel function for MFG-E8 in neuroprotection by microglia.

$\mathrm{oA} \beta$ is toxic to neuronal cells in the present study. It has been reported that $A \beta$ also enhances microglial activation to produce pro-inflammatory molecules [37-40]. $\mathrm{oA} \beta$ induces microglia to have a pro-inflammatory character [37], and increases microglial TNF- $\alpha$ secretion through a tyrosine kinase dependent pathway [39]. NOmediated microglial neurotoxicity by $\mathrm{oA} \beta$ is reported [40]. oA $\beta$ disturbs microglial phagocytosis and clearance of fibrillar $\mathrm{A} \beta$ [15].

However, oA $\beta$ did not induce inflammatory molecules, such as TNF- $\alpha$, NO and glutamate in microglia in the present and previous studies [24]. The synthetic oA $\beta$ used in the present study contains oligomers of different sizes and shapes, so that microglia may respond to this heterogeneous oA $\beta$ in various ways. Furthermore, synthetic $\mathrm{oA} \beta$ is less potent than $\mathrm{oA} \beta$ isolated from the transfected cell cultures.

Since MFG-E8 is a ligand for $\alpha_{v} \beta_{3}$ and $\alpha_{v} \beta_{5}$ integrins, MFG-E8 presumably interacts with microglia by binding to integrins [1,41]. MFG-E8 forms a cellular bridge between the integrin on phagocytes and PS on target cells. MFG-E8 induces CD47 expression in microglia. CD47 directly interacts with $A \beta$ to form a complex that induces microglial engulfment [29], after which MFG-E8 increases $A \beta$ clearance via CD47. RAGE is a surface molecule that directly interacts with $A \beta$ and activates NFkB-mediated signaling pathways in microglia $[42,43]$. Recent reports showed that RAGE also directly interacts with PS and enhances macrophage phagocytosis [44]. RAGE simultaneously enhances the inflammatory status. On the other hand, MFG-E8 enhances microglial phagocytosis without inducing inflammation.

Our data are schematically summarized in Figure 6. Glutamate and oA $\beta$ damage neurons, which then release soluble factors, including FKN, as "help-me" signals. In response to these factors, microglia release MFG-E8. MFG-E8 directly increases $A \beta$ phagocytosis by microglia via $\mathrm{CD} 47$ expression and also increases $\mathrm{HO}-1$ expression in microglia via nuclear translocation of the transcription factor Nrf2. Each of these processes contributes to microglial neuroprotection. Therefore, MFG-E8 or agents that increase its expression may be potential therapeutic targets for neurodegenerative diseases, including AD.

\section{Conclusions}

Here we show that MFG-E8 is released from microglia in response to soluble factors (for example, FKN) secreted from degenerated neurons. MFG-E8 induces microglial neuroprotective activity against oA $\beta$ toxicity. This neuroprotection is mediated through enhanced microglial phagocytic activity via the CD47 pathway and progression of the Nrf2-HO-1 pathway. These data indicate that MFG-E8 may have novel roles as a neuroprotectant that is produced from microglia during neurodegenerative conditions.

\section{Abbreviations}

AD: Alzheimer's disease; CNS: Central nervous system; FKN: Fractalkine; GAPDH: Glyceraldehydes-3-phosphate dehydrogenase; HO-

1: Hemeoxygenase-1; LPS: Lipopolysaccharide; MFG-E8: Milk fat globule-EGF factor 8; NO: Nitric oxide; NQO-1: NAD(P)H quinine oxidoreductase-1; Nrf2: Nuclear factor E(2)-related factor 2; oA $\beta$ : Oligomeric amyloid $\beta$; PS: Phosphatidylserine; RAGE: Receptor for advanced glycation end products; ROS: Reactive oxygen species; TLRs: Toll-like receptors; TNF-a: Tumor necrosis factor-a.

\section{Competing interests}

The authors declare that they have no competing interests.

\section{Acknowledgements}

This work was supported by the Program for Promotion of Fundamental Studies in Health Sciences from the National Institute of Biomedical Innovation (NIBIO); a Grant-in-Aid for the global COE program from the Ministry of Education, Culture, Sports, Science and Technology of Japan. Research Grant on Intractable Diseases (Neuroimmunological Diseases) from the Ministry of Health, Labour and Welfare of Japan. Research Grant from Hayashi Memorial Foundation for Female Natural Scientists.

\section{Authors' contributions}

EL performed the RT-PCR experiments and helped draft the manuscript. MN conducted the ELISAs, microglial phagocytosis assay and FACS analysis, and drafted the manuscript. YD, BP, JK and HT performed the cell culture and were involved in the conception of the study. TM carried out the immunocytochemistry. He was also involved in the conception and design of the study, and helped draft the manuscript. AS was also involved in the conception and design of the study, as well as in the preparation of the manuscript. All authors read and approved the final manuscript.

Received: 23 February 2012 Accepted: 1 May 2012

Published: 28 June 2012

\section{References}

1. Hanayama R, Tanaka M, Miwa K, Shinohara A, Iwamatsu A, Nagata S: Identification of a factor that links apoptotic cells to phagocytes. Nature 2002, 417:182-187.

2. Miyasaka K, Hanayama R, Tanaka M, Nagata S: Expression of milk fat globule epidermal growth factor 8 in immature dendritic cells for engulfment of apoptotic cells. Eur J Immunol 2004, 34:1414-1422. 
3. Noda M, Doi Y, Liang J, Kawanokuchi J, Sonobe Y, Takeuchi H, Mizuno T, Suzumura A: Fractalkine attenuates excito-neurotoxicity via microglial clearance of damaged neurons and antioxidant enzyme heme oxygenase-1 expression. J Biol Chem 2011, 286:2308-2319.

4. Boddaert J, Kinugawa K, Lambert JC, Boukhtouche F, Zoll J, Merval R, Blanc-Brude O, Mann D, Berr C, Vilar J, Garabedian B, Journiac N, Charue D, Silvestre JS, Duyckaerts C, Amouyel P, Mariani J, Tedgui A, Mallat Z: Evidence of a role for lactadherin in Alzheimer's disease. Am J Pathol 2007, 170:921-929.

5. Kranich J, Krautler NJ, Falsig J, Ballmer B, Li S, Hutter G, Schwarz P, Moos R, Julius C, Miele G, Aguzzi A: Engulfment of cerebral apoptotic bodies controls the course of prion disease in a mouse strain-dependent manner. J Exp Med 2010, 207:2271-2281.

6. Aziz MM, Ishihara S, Mishima Y, Oshima N, Moriyama I, Yuki T, Kadowaki Y, Rumi MA, Amano Y, Kinoshita Y: MFG-E8 attenuates intestinal inflammation in murine experimental colitis by modulating osteopontindependent $\alpha_{v} \beta_{3}$ integrin signaling. J Immunol 2009, 182:7222-7232.

7. Bu HF, Zuo XL, Wang X, Ensslin MA, Koti V, Hsueh W, Raymond AS, Shur BD, Tan XD: Milk fat globule-EGF factor 8/lactadherin plays a crucial role in maintenance and repair of murine intestinal epithelium. $J$ Clin Invest 2007, 117:3673-3683.

8. Chogle A, Bu HF, Wang X, Brown JB, Chou PM, Tan XD: Milk fat globuleEGF factor 8 is a critical protein for healing of dextran sodium sulfateinduced acute colitis in mice. Mol Med 2011, 17:502-507.

9. Farfara D, Lifshitz V, Frenkel D: Neuroprotective and neurotoxic properties of glial cells in the pathogenesis of Alzheimer's disease. J Cell Mol Med 2008, 12:762-780.

10. Neumann H, Kotter MR, Franklin RJ: Debris clearance by microglia: an essential link between degeneration and regeneration. Brain 2009, 132:288-295.

11. Alarcón R, Fuenzalida C, Santibáñez M, von Bernhardi R: Expression of scavenger receptors in glial cells. Comparing the adhesion of astrocytes and microglia from neonatal rats to surface-bound $\beta$-amyloid. J Biol Chem 2005, 280:30406-30415.

12. Landreth GE, Reed-Geaghan EG: Toll-like receptors in Alzheimer's disease Curr Top Microbiol Immunol 2009, 336:137-153

13. Hirt UA, Leist M: Rapid, noninflammatory and PS-dependent phagocytic clearance of necrotic cells. Cell Death Differ 2003, 10:1156-1164.

14. Reed-Geaghan EG, Reed QW, Cramer PE, Landreth GE: Deletion of CD14 attenuates Alzheimer's disease pathology by influencing the brain's inflammatory milieu. J Neurosci 2010, 30:15369-15373.

15. Pan XD, Zhu YG, Lin N, Zhang J, Ye QY, Huang HP, Chen XC: Microglial phagocytosis induced by fibrillar $\beta$-amyloid is attenuated by oligomeric $\beta$ amyloid: implications for Alzheimer's disease. Mol Neurodegener 2011, 6:45.

16. Persaud-Sawin DA, Banach L, Harry GJ: Raft aggregation with specific receptor recruitment is required for microglial phagocytosis of $A \beta 42$. Glia 2009, 57:320-335.

17. Koning N, Uitdehaag BM, Huitinga I, Hoek RM: Restoring immune suppression in the multiple sclerosis brain. Prog Neurobio/ 2009, 89:359-368.

18. Gitik M, Liraz-Zaltsman S, Oldenborg PA, Reichert F, Rotshenker S: Myelin down-regulates myelin phagocytosis by microglia and macrophages through interactions between CD47 on myelin and SIRPa (signal regulatory protein-a) on phagocytes. J Neuroinflammation 2011, 8:24

19. Bamberger ME, Harris ME, McDonald DR, Husemann J, Landreth GE: A cell surface receptor complex for fibrillar $\beta$-amyloid mediates microglial activation. J Neurosci 2003, 23:2665-2674.

20. McDonald DR, Brunden KR, Landreth GE: Amyloid fibrils activate tyrosine kinase-dependent signaling and superoxide production in microglia. J Neurosci 1997, 17:2284-2294.

21. Johnstone $M$, Gearing AJ, Miller KM: A central role for astrocytes in the inflammatory response to $\beta$-amyloid; chemokines, cytokines and reactive oxygen species are produced. J Neuroimmunol 1999, 93:182-193.

22. Yasui Y, Sasao E, Sakata M, Matsui N, Fukuishi N, Akagi R, Akagi M: Upregulation of heme oxygenase-1 by degranulation in rat basophilic leukemia cells. Biol Pharm Bull 2007, 30:443-446.

23. Li Q, Li J, Zhang L, Wang B, Xiong L: Preconditioning with hyperbaric oxygen induces tolerance against oxidative injury via increased expression of heme oxygenase- 1 in primary cultured spinal cord neurons. Life Sci 2007, 80:1087-1093.

24. Doi Y, Mizuno T, Maki Y, Jin S, Mizoguchi H, Ikeyama M, Doi M, Michikawa M, Takeuchi H, Suzumura A: Microglia activated with the toll-like receptor 9 ligand CpG attenuate oligomeric amyloid $\beta$ neurotoxicity in in vitro and in vivo models of Alzheimer's disease. Am J Pathol 2009, 175:2121-2132.

25. Banno M, Mizuno T, Kato H, Zhang G, Kawanokuchi J, Wang J, Kuno R, Jin S, Takeuchi $\mathrm{H}$, Suzumura A: The radical scavenger edaravone prevents oxidative neurotoxicity induced by peroxynitrite and activated microglia. Neuropharmacology 2005, 48:283-290.

26. Suzumura A, Mezitis SG, Gonatas NK, Silberberg DH: MHC antigen expression on bulk isolated macrophage-microglia from newborn mouse brain: induction of la antigen expression by $\gamma$-interferon. J Neuroimmunol 1987, 15:263-278.

27. Pollock JS, Forstermann U, Mitchell JA, Warner TD, Schmidt HH, Nakane M, Murad F: Purification and characterization of particulate endotheliumderived relaxing factor synthase from cultured and native bovine aortic endothelial cells. Proc Natl Acad Sci U S A 1991, 88:10480-10484.

28. Takeuchi H, Mizuno T, Zhang G, Wang J, Kawanokuchi J, Kuno R, Suzumura A: Neuritic beading induced by activated microglia is an early feature of neuronal dysfunction toward neuronal death by inhibition of mitochondrial respiration and axonal transport. J Biol Chem 2005, 280:10444-10454.

29. Koenigsknecht J, Landreth G: Microglial phagocytosis of fibrillar $\beta$-amyloid through a $\beta 1$ integrin-dependent mechanism. J Neurosci 2004, 24:9838-9846.

30. Chung J, Gao AG, Frazier WA: Thrombspondin acts via integrin-associated protein to activate the platelet integrin $\alpha_{11 b} \beta_{3}$. J Biol Chem 1997 272:14740-14746.

31. Hanayama R, Tanaka M, Miyasaka K, Aozasa K, Koike M, Uchiyama Y, Nagata S: Autoimmune disease and impaired uptake of apoptotic cells in MFG-E8-deficient mice. Science 2004, 304:1147-1150.

32. Fuller AD, Van Eldik LJ: MFG-E8 regulates microglial phagocytosis of apoptotic neurons. J Neuroimmune Pharmacol 2008, 3:246-256.

33. Komura H, Miksa M, Wu R, Goyert SM, Wang P: Milk fat globule epidermal growth factor-factor VIII is down-regulated in sepsis via the lipopolysaccharide-CD14 pathway. J Immuno/ 2009, 182:581-587.

34. Ray B, Chauhan NB, Lahiri DK: Oxidative insults to neurons and synapse are prevented by aged garlic extract and S-allyl-L-cysteine treatment in the neuronal culture and APP-Tg mouse model. J Neurochem 2011, 117:388-402.

35. Newington JT, Pitts A, Chien A, Arseneault R, Schubert D, Cumming RC: Amyloid $\beta$ resistance in nerve cell lines is mediated by the Warburg effect. PLoS One 2011, 6:e19191.

36. De Felice FG, Velasco PT, Lambert MP, Viola K, Fernandez SJ, Ferreira ST, Klein WL: Abeta oligomers induce neuronal oxidative stress through an $\mathrm{N}$-methyl-D-aspartate receptor-dependent mechanism that is blocked by the Alzheimer drug memantine. J Biol Chem 2007, 282:11590-11601.

37. Michelucci A, Heurtaux T, Grandbarbe L, Morga E, Heuschling P: Characterization of the microglial phenotype under specific proinflammatory and anti-inflammatory conditions: Effects of oligomeric and fibrillar amyloid- $\beta$. J Neuroimmunol 2009, 210:3-12.

38. Mandrekar-Colucci S, Landreth GE: Microglia and inflammation in Alzheimer's disease. CNS Neurol Disord Drug Targets 2010, 9:156-167.

39. Dhawan G, Floden AM, Combs CK: Amyloid- $\beta$ oligomers stimulate microglia through a tyrosine kinase dependent mechanism. Neurobiol Aging 2011, [Epub ahead of print].

40. Maezawa I, Zimin PI, Wulff $H$, Jin LW: Amyloid- $\beta$ protein oligomer at low nanomolar concentrations activates microglia and induces microglial neurotoxicity. J Biol Chem 2011, 286:3693-3706.

41. Nandrot EF, Anand M, Almeida D, Atabai K, Sheppard D, Finnemann SC: Essential role for MFG-E8 as ligand for $\alpha_{v} \beta_{5}$ integrin in diurnal retinal phagocytosis. Proc Natl Acad Sci U S A 2007, 104:12005-12010.

42. Yan SD, Chen X, Fu J, Chen M, Zhu H, Roher A, Slattery T, Zhao L, Nagashima M, Morser J, Migheli A, Nawroth P, Stern D, Schmidt AM: RAGE and amyloid- $\beta$ peptide neurotoxicity in Alzheimer's disease. Nature 1996, 382:685-691.

43. Yan SD, Stern D, Kane MD, Kuo YM, Lampert HC, Roher AE: RAGE-A $\beta$ interactions in the pathophysiology of Alzheimer's disease. Restor Neurol Neurosci 1998, 12:167-173.

44. Friggeri A, Banerjee S, Biswas S, de Freitas A, Liu G, Bierhaus A, Abraham E: Participation of the receptor for advanced glycation end products in efferocytosis. J Immunol 2011, 186:6191-6198.

doi:10.1186/1742-2094-9-148

Cite this article as: Li et al:: The neuroprotective effects of milk fat globuleEGF factor 8 against oligomeric amyloid $\beta$ toxicity. Journal of Neuroinflammation 2012 9:148. 